\title{
Análisis del patrón espacio-temporal de transmisión del COVID-19 por municipios de Baja California
}

\section{Analysis of the spatio-temporal transmission pattern of COVID-19 by municipalities of Baja California}

Recibido el 5 de octubre de 2020.

Aceptado el 25 de mayo de 2021.

Publicado el 4 de junio de 2021.

*Autor para correspondencia: Alejandro Brugués Rodríguez. Correo electrónico: abrugues@colef.mx
Esta obra está protegida bajo una Licencia Creative Commons Atribución-NoComercial 4.0 Internacional.

\begin{abstract}
Alejandro Brugués Rodríguez ${ }^{\text {a* }}$ Noé Arón Fuentes Flores ${ }^{b}$ Alejandra Ramírez Cervantes ${ }^{c}$

https://orcid.org/0000-0001-5964-7974

https://orcid.org/0000-0002-9254-8107

https://orcid.org/0000-0001-7240-7414
\end{abstract}

a El Colegio de la Frontera Norte, Ciudad Juárez, México, correo electrónico: abrugues@colef.mx
b El Colegio de la Frontera Norte, Tijuana, México, correo electrónico: afuentes@colef.mx
' Universidad Autónoma de Baja California, Facultad de Economía y Relaciones Internacionales,
Tijuana, México, correo electrónico: aramirez17@uabc.edu.mx

Resumen

El texto analiza del patrón espacio-temporal de propagación del COVID-19 en los municipios de Baja California desde la semana epidemiológica 10 hasta la 31 con base en las metodologías de Dinámica de Sistemas (DS) y Sistemas de Información Geográfica (sIG). Se usa el modelo susceptibles, infectados y recuperados (SIR) epidémico a fin de modelar los factores críticos de contagio de la pandemia - tasa de infección y tasa de recuperación de la infeccióncon datos de la Dirección General de Epidemiología de la Secretaría de Salud disponibles el día 6 de junio de 2020. El patrón epidemiológico tiende a concentrase espacialmente en Mexicali, Tijuana y Tecate, ciudades que albergan a los trabajadores transfronterizos entre las Californias. Además, presenta una dinámica temporal cambiante hacia los municipios de Ensenada y Playas de Rosarito que son el destino de mayor demanda de los turistas californianos.

Palabras clave: Patrón de transmisión, COVID-19, municipios de Baja California.

\section{Abstract}

The text analyzes the spatio-temporal pattern of spread of COVID-19 in the municipalities of Baja California from epidemiological week 10 to 31 based on System Dynamics (SD) and Geographic Information Systems (GIS) methodologies. The epidemic sIR model is used to model the critical factors of pandemic congestion -infection rate and infection recovery rate- with data from the General Directorate of Epidemiology of the Ministry of Health available on June 6, 2020. The epidemiological pattern tends to be spatially concentrated in Mexicali, Tijuana

CÓMO CITAR: Brugués Rodríguez, A., Fuentes Flores, N. A. \& Ramírez Cervantes, A. (2021). Análisis del patrón espacio-temporal de transmisión del COVID-19 por municipios de Baja California [Analysis of the spatio-temporal transmission pattern of COVID-19 by municipalities of Baja California] Estudios Fronterizos, 22, e071. https://doi.org/10.21670/ref.2108071 
and Tecate, which are home to cross-border workers between the Californias. In addition, it presents a changing temporal dynamic towards the municipalities of Ensenada and Playas de Rosarito, which are the most demanded destinations by Californian tourist.

Keywords: Transmission pattern, COVID-19, Baja California municipalities.

\section{Introducción}

A medida que el SARS-CoV-2 (COVID-19) se propaga en México se van conociendo más características de su dinámica de contagios entre las entidades federativas. La determinación del patrón de infecciones en el espacio y tiempo ayuda a entender cómo ocurre la propagación y la forma en que se transmite en medio de las acciones de control establecidas por los sistemas de vigilancia epidemiológica y, en consecuencia, auxilia a redefinir las estrategias de intervención para disminuir el impacto en la salud de las poblaciones (Bhaskar et al., 2020; Cuartas et al., 2020; Medeiros et al., 2020; Parr, 2020). Este texto tiene como objetivo analizar el patrón espacio-temporal de propagación del COVID-19 en los municipios de la entidad fronteriza de Baja California entre la semana epidemiológica 10 y la 31 por medio de la utilización de las metodologías de Dinámica de Sistemas (SD, por sus siglas en inglés) y Sistemas de Información Geográfica (sIG). Estas herramientas pueden simular la complejidad y multiplicidad de aspectos involucrados en este tipo de fenómeno epidemiológico (Castro et al., 2005).

Para modelar los factores críticos de la propagación del COVID-19, como son la tasa de contagio y la tasa de recuperación de la infección, se empleó el modelo siR epidémico (Kermack \& McKendrick, 1927) que ha sido ampliamente utilizado en México (Miramontes, 2020; Ortigoza et al., 2020; Ruiz, 2020; Vargas Magaña et al., 2020). La información sobre los casos positivos acumulados por semana proviene de la Dirección General de Epidemiología de la Secretaría de Salud (DGE-ss) registrados el 6 de junio de 2020 .

El estado de Baja California, el día 6 de junio de 2020, rebasó la cifra de 6000 casos positivos acumulados de COVID-19 con lo que ocupó el tercer lugar en el nivel nacional (DGE-ss, 2020). El patrón de contagios por municipios muestra que Mexicali es el principal foco, al llegar a 3079 —la mitad de pacientes a nivel estatal—, Tijuana llegó a 2 276, Ensenada tuvo 312, Tecate 180 y Playas de Rosarito 64 (DGE-ss, 2020). En cuanto al número acumulado de muertes por la enfermedad está liderado por Tijuana con 643 decesos, de ahí sigue Mexicali con 495, Ensenada con 51, Tecate con 50 y Playas de Rosarito con 9. Adicionalmente, es importante destacar que esta entidad federativa al compararla con las cifras nacionales tiene una tasa de mortalidad estatal de 2.36 por cada 100000 habitantes, muy por encima de la media nacional que es de 0.076 por cada 100000 habitantes y una tasa de letalidad de $8.7 \%$, también superior a la media nacional de $4.39 \%$ (DGE-Ss, 2020).

Los resultados muestran que el patrón epidemiológico tiende a concentrase espacialmente en Mexicali, Tijuana y Tecate, ciudades que albergan a los trabajadores transfronterizos entre las californias. Además, presenta una dinámica temporal cambiante hacia los municipios de Ensenada y Playas de Rosarito que son el destino de mayor demanda de los turistas californianos. 
A continuación, el texto se divide en siete secciones. En la primera se presenta una revisión de la literatura sobre la propagación del COVID-19 en México. En la segunda se presenta el modelo sir que es denominado epidémico puesto que la duración de la enfermedad es corta comparada con la esperanza de vida del huésped (Pérez, 2012). En la tercera se presenta la metodología de SD, útil para analizar las causas estructurales que provocan el comportamiento dinámico de modelo sIR y el software Stella es una herramienta que permite ejecutar simulaciones numéricas del modelo sIR en computadora. En la cuarta sección se presenta la metodología sIG, útil para representar geográficamente la evolución del COVID-19 en el estado. En la quinta parte se presenta la base de datos. En la sexta se discuten los resultados de la evolución geográfica-temporal de la transmisión del COVID-19 en los cinco municipios de la entidad fronteriza de Baja California. Y finalmente, en la séptima sección se concluye.

\section{Propagación del COVID-19 en México}

En México, el primer caso confirmado de COVID-19 se reportó el 27 de febrero de 2020, de una persona que estuvo de viaje en Italia, y el primer deceso no importado del virus ocurrió el 19 de marzo. Para la primera fecha, el país se encontraba en la denominada Fase 1, donde los casos positivos del virus en México se asociaban a personas que viajaron contagiadas procedentes de países con presencia de la enfermedad. Entre el 17 y el 23 de marzo en el país, basados en el principio de reducción del número de contactos, se aumentó la cantidad e intensidad de acciones de mitigación no farmacológicas, tales como la cancelación de las actividades escolares, el uso del distanciamiento social, el lavado de manos, el no saludar de beso o abrazo y la no celebración de reuniones con más de 50 personas. Dos días después —el 25 de marzo — se consideró declarar la Fase 2, por la existencia de contagios comunitarios en personas sin antecedentes de viaje al extranjero. Poco menos de un mes después —el 21 de abril— se declara la entrada de México a la Fase 3 que extiende la Jornada de Sana Distancia, así como la suspensión de actividades, hasta el 30 de mayo (Palacios, 2020).

En el país se ha aplicado el modelo sir epidémico, originalmente desarrollado por Kermack y McKendrick (1927), a fin de determinar temporalmente cuándo surge el brote de infección del COVID-19, cómo crece, en qué momento alcanza su máximo y cómo después decrece (Miramontes, 2020; Ortigoza et al., 2020; Ruiz, 2020; Vargas Magaña et al., 2020).

Así, Ruiz (2020) realizó una simulación de la infección basado en el modelo sIR epidémico bajo ciertos supuestos: 1) El total de personas en el país es constante en 120 millones con lo que prescinde de la dinámica vital. 2) Cualquier mexicano es susceptible de contagiarse del virus, excepto aquellos que se han recuperado. 3) La propagación del virus en el país se originó a partir de una sola persona. 4) El virus tiene un periodo infeccioso de 14 días, correspondientes a una tasa de recuperación diaria de la infección $y=14^{-1}=0.072$ dias $^{-1} \mathrm{y}$ una tasa diaria de infección $\beta=0.145$. Y, 5) Las condiciones iniciales son: $S_{0}=0.99, \mathrm{I}_{0}=0.01$ Estos datos implican una razón de reproducción de la infección $R_{0}=2.2$.

De la simulación de la propagación del COVID-19, el autor concluye que, en promedio, cada persona infectada era capaz de infectar a 2.2 personas, que el máximo 
de población contagiada sería del 12\% y señala que después de 365 días la epidemia del COVID-19 habría de terminar, contados desde el primer caso ocurrido el día 27 de febrero de 2020.

En tanto, Vargas Magaña y colaboradores consideran que si bien el modelo sIR asume al parámetro $\beta$ fijo durante la evolución de la pandemia debido a la aplicación de políticas sanitarias antiepidémicas en el país, se puede deducir que tiende a reducir su valor temporalmente, y consecuentemente afectar la tasa de reproducción básica de la infección en el tiempo. De esta manera, analizan cómo las medidas de mitigación de la epidemia afectan la evolución del número promedio diario de infectados $\beta(t)$ en el tiempo. O alternativamente, cómo se afectó la velocidad del contagio de la enfermedad infecciosa $R(t)$ en el tiempo.

Los autores señalan que las autoridades sanitarias mexicanas instrumentaron en total tres fases sanitarias, cada una según el grado de transmisión de la enfermedad. Cada una de estas fases contribuyeron a reducir el valor de $\beta$ y consecuentemente al descenso del valor de $R_{0}$ de 2.409 a 1.391 . En la Tabla 1 podemos ver el efecto de mitigación o "aplanamiento" del brote inicial de COVID-19 según los parámetros $\beta, y, \mathrm{y}, R_{0}$.

Tabla 1. Evolución del COVID-19 con el modelo sIR con $\beta$ variable

\begin{tabular}{|c|c|c|c|}
\hline Estimaciones & $\boldsymbol{\beta}$ & $\boldsymbol{y}$ & $\boldsymbol{R}_{\boldsymbol{0}}$ \\
\hline Fase 1, semana 2 & 0.241 & 0.100 & 2.40 \\
\hline Fase 2, semana 3 & 0.210 & 0.099 & 2.10 \\
\hline Fase 2, semana 5 & 0.170 & 0.100 & 1.79 \\
\hline Fase 3, semana 6 & 0.169 & 0.099 & 1.69 \\
\hline Fase 3, semana 8 & 0.129 & 0.100 & 1.29 \\
\hline Fase 3, semana 10 & 0.139 & 0.099 & 1.39 \\
\hline
\end{tabular}

Fuente: Vargas Magaña et al., 2020

De las simulaciones de la epidemia infieren que la evolución decreciente del valor $R(t)$ es señal de un aplanamiento exitoso de la curva de contagio que es el resultado, a su vez, de una baja movilidad, del aislamiento, de la suspensión de ciertas actividades económicas, de la restricción de congregaciones masivas y de la sana distancia, entre otras. En ausencia de la aplicación de una vacuna, las anteriores medidas fueron una solución para aplanar la curva de infecciones.

Miramontes (2020) también analiza la evolución del número de personas infectadas en distintos escenarios, en donde la diferencia se produce exclusivamente en la variación de la tasa de transmisión de la infección promedio diaria $\beta(t)$ y, por tanto, en la incertidumbre de la determinación de la tasa reproductiva básica $\left(R_{0}\right)$. Establece que varios estudios a nivel internacional señalan un valor promedio de $R_{0}=3.28$, pero la incertidumbre era muy alta, al ubicar el valor del coeficiente en un intervalo de entre 1.50 y 6.68 (Viceconte \& Petrosillo, 2020 citados en Miramontes, 2020).

Por último, Ortigoza y colaboradores simulan numéricamente un modelo siR epidémico aplicado al COVID-19 para el estado de Veracruz con el empleo de datos semanales. Para ello, se requirió ajustar el modelo sir a los datos oficiales mediante estimaciones de los parámetros y y $\beta$ con el uso de una regresión estimada entre la subpoblación de infectados oficiales y el número de infectados ajustados de un modelo 
SIR inicial. Para la simulación numérica del modelo se usó el paquete Matlab 2017a. Se asumió una población total de 8112505 individuos con condiciones iniciales de la infección del COVID-19 específicas de la entidad: $S_{0}=8112504, I_{(0)}=7$ y $R_{(0)}=0$.

Los resultados obtenidos de los parámetros son $y=0.69$ y $\beta=1$.33. Estos implican una razón de $R_{0}=1.93$, es decir, cada persona infectada era capaz de infectar a 1.93 personas.

En la Figura 1 se replica la variación de las poblaciones: sanos, infectados y recuperados, involucradas en Veracruz al usar el modelo sIR epidémico con respecto al tiempo, debido a la dinámica sistémica de las interacciones.

Figura 1. Evolución de sanos, infectados y recuperados de COVID-19 de Veracruz

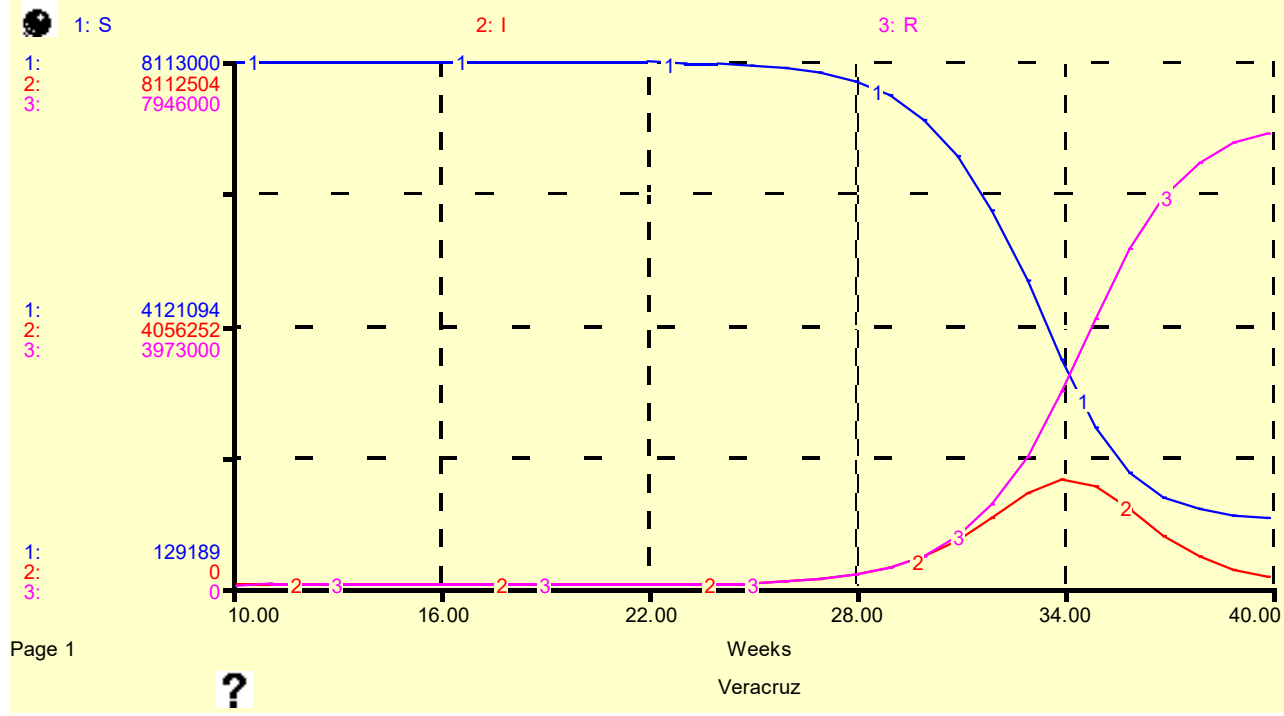

Fuente: Estimación propia basada en los parámetros de Ortigoza y colaboradores, 2020

Nota: 1.s, sanos; 2.I, infectados; y, 3.R, recuperados

De la simulación numérica se identifica que el pico máximo de infectados en Veracruz se alcanza en la semana 33.

En síntesis, el modelo epidemiológico sIR se ha aplicado en el país y entidades para la comprensión de la evolución y del efecto de las medidas de contención del COVID-19.

\section{Modelo susceptibles, infectados y recuperados (SIR)}

El modelo SIR epidémico emplea sistemas de ecuaciones diferenciales aunadas a ciertos elementos de abstracción para describir, explicar y simular el comportamiento de una enfermedad que se propaga por contagio en una población que permanece constante. También se usa como herramienta para tomar decisiones que permitan evaluar y definir la mejor estrategia de mitigación, prevención o control para limitar el impacto económico o sanitario que genera una enfermedad. Asimismo, sirve 
como instrumento de análisis que permite mejorar la percepción de las dinámicas que presentan las enfermedades contagiosas, y así validar la relación de los sistemas predictores con la ocurrencia de la enfermedad, principalmente en relación con el manejo de la razón de reproducción básica de la infección $\left(R_{0}\right)$, que debe ser considerado para el control y la erradicación de la enfermedad (García Piñera, 2014).

Originalmente Kermack y McKendrick (1927) formularon el modelo a partir de los postulados siguientes: 1) la enfermedad puede ser de carácter viral o bacteriana y es transmitida por contacto directo de persona a persona; 2) en el origen de la epidemia únicamente una fracción de la población era contagiosa; 3) la población es cerrada y con excepción de los pocos individuos inicialmente infectados todas las demás son susceptibles de enfermarse; 4) la enfermedad tiene un ciclo de tres estados: susceptibles (s), infectados (I) y recobrados (R); 5) el enfermo sufre el ciclo completo de la enfermedad para al final recuperarse y adquirir inmunidad; y, G) la población es constante o sin dinámica vital.

Al modelo sIR epidémico siguiente se le considera epidémico puesto que la duración de la enfermedad es corta comparada con la esperanza de vida del huésped (Pérez, 2012). Razón por la cual los nacimientos y muertes no son considerados, es decir, se considera que la población es constante.

$$
\begin{aligned}
& \frac{\mathrm{dS}}{\mathrm{dt}}=-\beta S I \\
& \frac{\mathrm{dI}}{\mathrm{dt}}=\beta S I-y I \\
& \frac{\mathrm{dR}}{\mathrm{dt}}=y I
\end{aligned}
$$

Donde las variaciones de los subgrupos de poblaciones consideradas se describen por el sistema de ecuaciones [1] a [3] y los parámetros $\beta$ y y denotan las tasas de infección y de recuperación, respectivamente.

Al partir de número inicial de infectados $\left(I_{(0)}\right)$ las condiciones poblacionales iniciales requeridas en el modelo son las siguientes,

$$
N=S_{(0)}-I_{(0)}, I_{(0)}=I_{0}, \mathrm{y} \mathrm{R}_{(0)}=0
$$

En el modelo epidémico se define al número de reproducción básico $\left(R_{0}\right)$ como:

$$
R_{0}=\frac{\beta}{y}
$$

así el valor de $R_{0}$ dependerá de las características epidemiológicas de la enfermedad reflejadas en los parámetros $\beta, y$, y de la población total N. Por tanto, $R_{0}$ tomará un valor constante (García Piñera, 2014).

La importancia de parámetro $R_{0}$ radica en que con base en él se pueda afirmar si existe brote epidémico cuando se realiza un análisis cualitativo de los modelos anteriores, observándose si

$$
R_{0}>1
$$

habrá un brote epidémico, y si

$$
R_{0}<1
$$


no habrá un brote epidémico.

En la práctica una forma de obtener información sobre $\beta, y$, y $R_{0}$ es simularlo numéricamente en el tiempo mediante SD.

\section{Metodología de Sistemas Dinámicos}

Uno de los enfoques para obtener información crítica del modelo visto es simularlo numéricamente en el tiempo mediante el método de SD que permita comprender las causas estructurales que provocan el comportamiento de cada sistema. Esto implica aumentar el conocimiento sobre el papel de cada elemento del sistema, y ver cómo diferentes acciones, efectuadas sobre partes del sistema, acentúan o atenúan las tendencias de comportamiento implícitas en cada uno (Delgado, 2017).

Para alcanzar el conocimiento de las causas estructurales que provocan el comportamiento de los sistemas, la metodología de SD se basa en la resolución de ecuaciones diferenciales con el empleo de una estructura basada en la descomposición de estas ecuaciones en tipos de variables, de stock o estado, y de flujo o transferencia; además, de variables auxiliares que permiten almacenar formulas o parámetros, y de arcos que representan los lazos causales.

Específicamente, se adoptó el software Stella que es una herramienta visual de modelaje que permite conceptualizar, documentar, simular, analizar y optimizar modelos de dinámica de sistemas. El software Stella provee una forma simple y flexible de construir modelos de simulación, basados en los diagramas de Forrester o diagramas de stock y flujos. Los elementos del diagrama son mostrados en la Figura 2.

Figura 2. Elementos del diagrama de Forrester

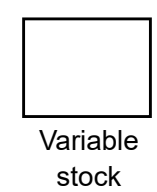

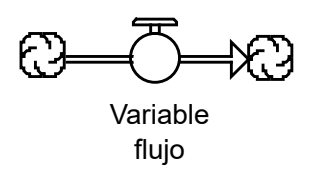

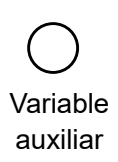

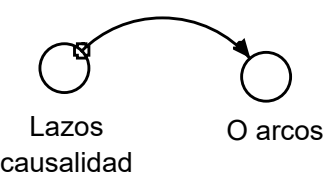

Fuente: Forrester, 1960

Así, el conjunto de variables de los modelos vistos se transforma en las siguientes:

Susceptibles (variable de estado) que representa la población total que no cuenta con defensas biológicas para rechazar el contagio de la enfermedad.

Infectados (variable de estado) que incluye a los enfermos contagiosos no controlados por los servicios de salud.

Recobrados o fallecidos (variable de estado) que son enfermos que se han curado, que quedan inmunizados o fallecen.

Nuevos contagios (variable flujo) que denota la tasa de infección.

Nuevos curados (variable flujo) que indica la tasa de recuperación.

En la Figura 3 se representa el modelo sIR epidémico en el diagrama de flujo de Forrester. 
Figura 3. Diagrama de flujo de Forrester para modelo sIR epidémico

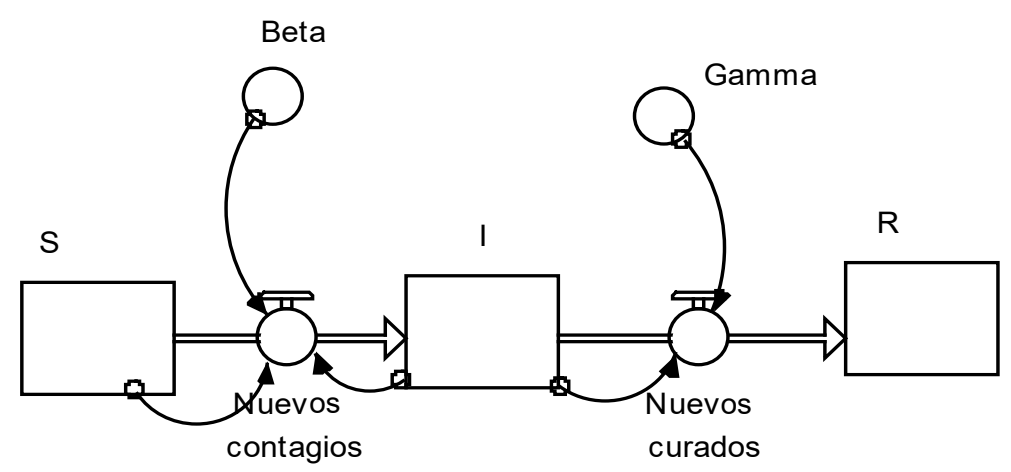

Fuente: Elaboración propia

En el diagrama de Forrester se muestra que la variable de nuevos contagios es un acumulador en el cual se suman, para cada nueva interacción, los sanos que se infectaron más los infectados de la interacción anterior. La variable de nuevos curados es un acumulador de los infectados que se recuperaron. Los datos de poblaciones se almacenan en las variables stock susceptibles o sanos (s), infectados (I) y recobrados (R). Por lo tanto, un infectado acumulado puede ser el resultado o de un sano o de un infectado en la interacción anterior.

Como resultado de la simulación se obtendrá la variación de las poblaciones de sanos, infectados y recobrados involucradas en el modelo con respecto al tiempo, debido a la Ds de las interacciones entre las variables de cada modelo.

\section{Metodología de Sistemas de Información Geográfica (SIG)}

Se puede retomar el modelo visto y la distribución de varias poblaciones espacialmente distribuidas para instrumentar y ejecutar la herramienta de SIG con el fin de visualizar e interpretar las fluctuaciones de sanos, infectados y recuperados por polígono en una zona de influencia (Castro et al., 2005).

La adecuación del modelo epidémico en el sıg permite realizar análisis cuantitativos sobre la distribución espacial por polígonos, realizar análisis de correlación espacial entre los datos y estudios de propagación de epidemia por métodos geográficos.

La metodología específica requiere de mapas de los polígonos de la región que contienen la información tabular y espacial de las poblaciones de sanos, infectados y recuperados por polígono en un tiempo inicial. Después se reclasifican los mapas iniciales, lo que implica a) implantar el modelo siR en la tabla de los datos espaciales; b) ajustar el rango de variación de las anteriores poblaciones de cada polígono; y, $c$ ) mediante el módulo de análisis espacial del sig clasificar los datos en intervalos de igual magnitud de tal manera que se pueda visualizar de una manera estándar y hacer análisis comparativos entre los mapas obtenidos. Y luego de la reclasificación se puede ejecutar el modelo bajo la plataforma del sig, para 20 interaciones. 
Como resultado se puede mostrar la distribución inicial y la variación de infectados por polígono en la zona de influencia de la pandemia por COVID-19 en los municipios de Baja California.

\section{Base de datos}

El análisis de la evolución del COVID-19 se realiza por municipio bajacaliforniano y para los condados fronterizos de California durante las semanas epidemiológicas 10 a 31 del año 2020. Los datos diarios se obtuvieron de la Dirección General de Epidemiología de la Secretaría de Salud (DGE-ss) en el caso de los municipios de México y de USAFacts.org en el caso de los condados de California. Una vez obtenida la base de información diaria se ha creado el número semanal de infectados, como se muestra en la Tabla 2.

Tabla 2. Datos de casos positivos reportados

\begin{tabular}{|c|c|c|c|c|c|c|c|}
\hline Semanas & Tijuana & Mexicali & Ensenada & Tecate & $\begin{array}{l}\text { Playas de } \\
\text { Rosarito }\end{array}$ & San Diego & Imperial \\
\hline 10 & 0 & 1 & 0 & 0 & 0 & 7 & 0 \\
\hline 11 & 4 & 4 & 0 & 0 & 0 & 72 & 2 \\
\hline 12 & 19 & 19 & 1 & 1 & 1 & 222 & 7 \\
\hline 13 & 123 & 63 & 4 & 2 & 2 & 552 & 34 \\
\hline 14 & 175 & 90 & 3 & 11 & 2 & 681 & 32 \\
\hline 15 & 242 & 193 & 12 & 14 & 8 & 961 & 42 \\
\hline 16 & 320 & 214 & 20 & 25 & 2 & 972 & 94 \\
\hline 17 & 310 & 235 & 43 & 28 & 11 & 941 & 101 \\
\hline 18 & 327 & 329 & 43 & 38 & 15 & 884 & 184 \\
\hline 19 & 260 & 454 & 42 & 26 & 8 & 956 & 268 \\
\hline 20 & 253 & 612 & 57 & 12 & 6 & 865 & 336 \\
\hline 21 & 201 & 691 & 67 & 13 & 6 & 843 & 464 \\
\hline 22 & 145 & 601 & 106 & 12 & 4 & 815 & 581 \\
\hline 23 & 133 & 690 & 112 & 19 & 2 & 1054 & 1358 \\
\hline 24 & 173 & 604 & 157 & 11 & 6 & 980 & 1092 \\
\hline 25 & 203 & 581 & 225 & 14 & 20 & 1774 & 1067 \\
\hline 26 & 230 & 601 & 205 & 28 & 23 & 3082 & 912 \\
\hline 27 & 341 & 542 & 215 & 12 & 26 & 3314 & 917 \\
\hline 28 & 204 & 413 & 204 & 22 & 14 & 3504 & 701 \\
\hline 29 & 259 & 332 & 220 & 24 & 10 & 3692 & 629 \\
\hline 30 & 243 & 246 & 209 & 21 & 13 & 3145 & 447 \\
\hline 31 & 242 & 244 & 162 & 32 & 21 & 2577 & 385 \\
\hline Población & 1789531 & 1087478 & 536143 & 113857 & 107859 & 3095313 & 174528 \\
\hline
\end{tabular}

Fuente: Elaboración directa con base en estadísticas de la DGE-SS (2020) y de USAFacts.org (2020) 
Se agrega la información de los condados de San Diego y el Valle Imperial de California, Estados Unidos, debido a la contigüidad y gran interacción en términos laborales y económicos con los municipios de Baja California.

\section{Distribución temporal-espacial del COVID-19 en municipios de Baja California}

Para ilustrar el ajuste temporal del modelo sIR epidémico aplicado al COVID-19 para al caso de Mexicali, Tijuana, Tecate, Ensenada y Playas de Rosarito se han utilizado datos semanales disponibles sobre el número de infectados por COVID-19 desde la semana epidemiológica 11 hasta la 31 . El proceso de obtención los de los valores de los parámetros $\beta$ y y requirió ajustar un modelo sIR inicial con la suposición de una tasa de contagio de 1.33 y una tasa de recuperación de 0.39 (Ortigoza et al., 2020). Posteriormente, se minimizó la suma de cuadrados residuales entre el número oficial de personas infectadas positivas y el número de personas infectadas a partir del modelo SIR ajustado (Ortigoza et al., 2020).

En la Tabla 3 se presentan los resultados obtenidos de los valores de $\beta$, y y $R_{0}$ para cada uno de los municipios de Baja California.

Tabla 3. Evolución del COVID-19 con el modelo sIR por municipio y $\beta$ fijo

\begin{tabular}{|l|c|c|c|}
\multicolumn{1}{|c|}{ Estimaciones } & $\boldsymbol{\beta}$ & $\boldsymbol{y}$ & $\mathbf{R}_{\mathbf{0}}$ \\
\hline Tijuana & 1.49 & 0.59 & 2.38 \\
\hline Mexicali & 1.60 & 0.65 & 2.46 \\
\hline Ensenada & 0.27 & 0.69 & 0.39 \\
\hline Tecate & 0.26 & 0.67 & 0.39 \\
\hline Playas de Rosarito & 0.28 & 0.70 & 0.40 \\
\hline
\end{tabular}

Fuente: Elaboración propia

La Tabla 3 presenta los factores críticos de la propagación inicial del COVID-19 municipal según el modelo durante las semanas epidemiológicas 10 a 31 de 2020. De los resultados se ve que Mexicali muestra la mayor tasa de contagios $\beta=1.60$. Mientras que Tijuana exhibe la menor tasa de recuperación $y=0.59$. Además, Mexicali muestra una razón de reproducción de la infección $R_{0}=2.46$ que es superior a la tasa nacional que varía entre $R_{0}=1.3$ y $R_{0}=2.4$.

En la Figura 4 se muestran las simulaciones del número de individuos susceptibles (1.s), infectados (2.I) y recobrados (3.R) de COVID-19 para Mexicali que es el de mayor contacto (por razones de espacio no se presentan las demás). 
Figura 4. Evolución de sanos, infectados y recuperados de COVID-19 para Mexicali

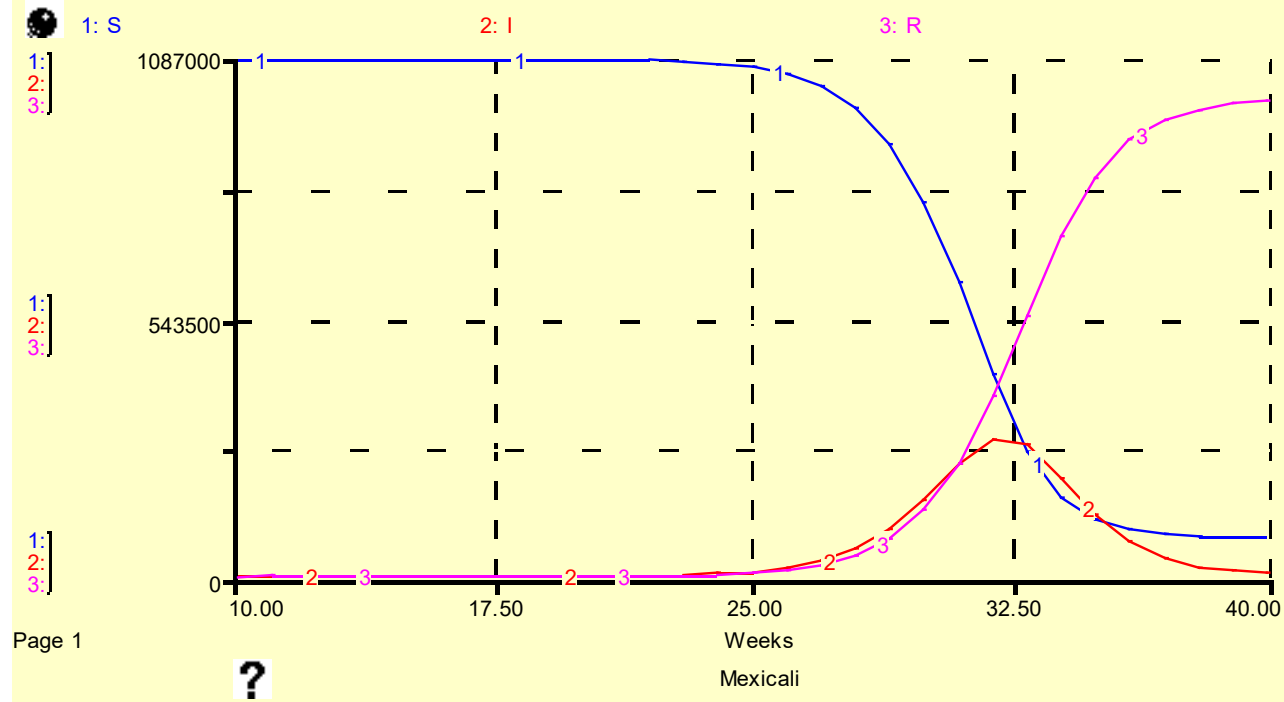

Fuente: Elaboración propia

Nota: 1.s, sanos, 2.I, infectados y 3.R, recuperados

En la Figura 4 se observan unas curvas típicas de infectados en la cual el pico de infectados se produce alrededor de la semana 31 para Mexicali.

Por otra parte, el análisis de los contagios desde una perspectiva espacial permite conocer no solo la localización, sino también permite incorporar al mismo las relaciones que tienen los contagios de una localización respecto a los contagios en otras localizaciones. En esta sección se fundamenta la explicación del comportamiento de la pandemia basado en la interacción de los residentes de los municipios de Baja California con los condados de San Diego y el Valle Imperial en California, Estados Unidos.

En la siguiente serie de mapas se aprecia cómo el inicio de la pandemia y su distribución espacial con la mayor cantidad de casos positivos por semana es el condado de San Diego, que mostró un crecimiento inicial durante las primeras cinco semanas, luego un periodo de 10 semanas de comportamiento estable y hacia el final, después de un crecimiento acelerado por cinco semanas, una ligera disminución en las dos semanas finales. En relación con el mismo, el comportamiento de los municipios de Tijuana, Tecate y Playas de Rosarito — geográficamente los más cercanos a San Diegomuestra también un crecimiento en las primeras semanas, aunque se observa hasta la semana 17, que es seguido por un espacio de cinco semanas donde disminuye y finaliza con un crecimiento sostenido. En Ensenada, con una muy importante interacción turística con San Diego y el resto de corredor costero que incluye a Tijuana y Rosarito hay un crecimiento sostenido durante las primeras 15 semanas para luego tener un comportamiento estable. 
Figura 5. Comportamiento espacio-temporal del COVID-19 en la región fronteriza de Baja California, México, y California, Estados Unidos

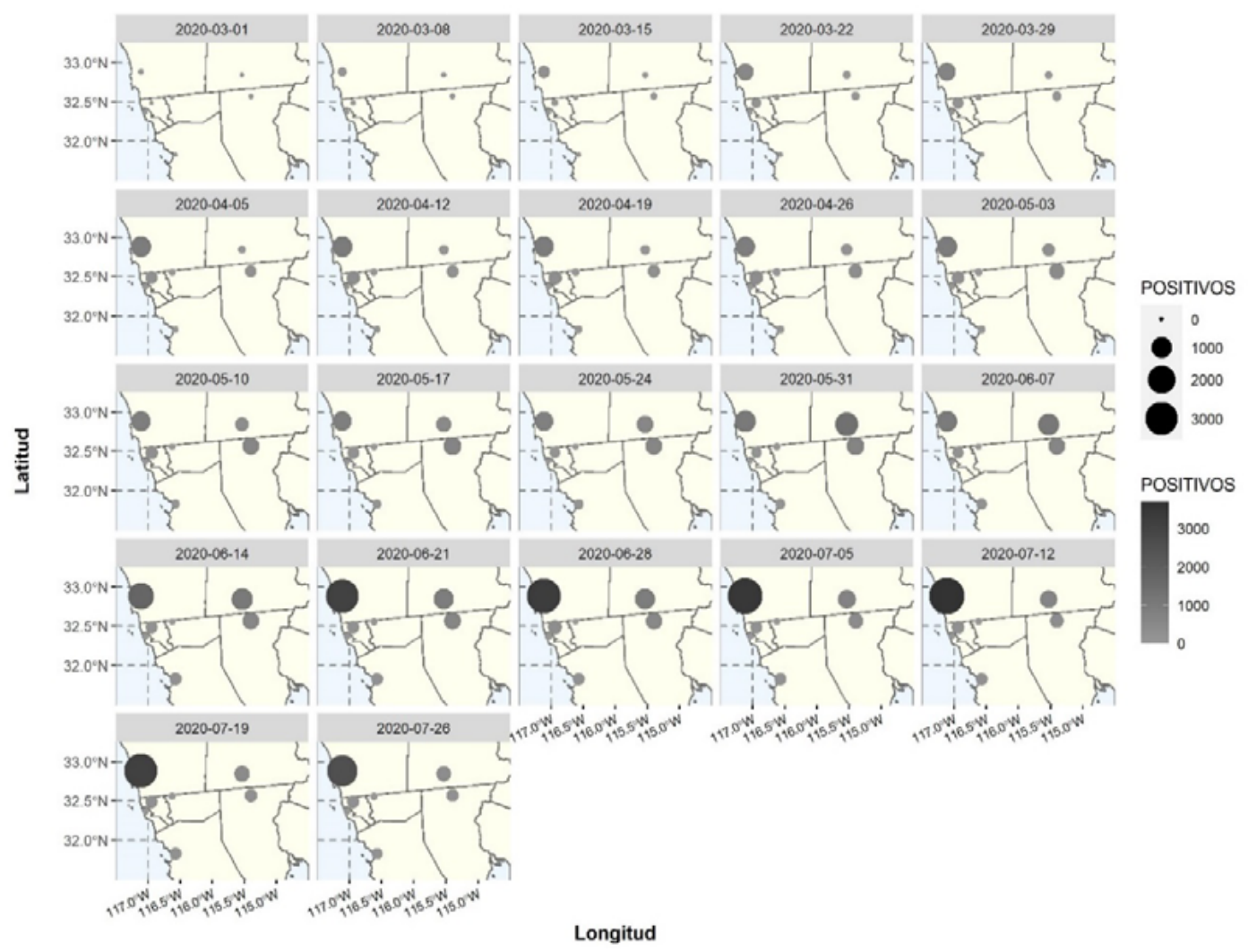

Fuente: Elaboración directa con base en estadísticas de la DGE-SS (2020) y de USAFacts.org (2020)

De la Figura 5 se observa que, al oeste de la región de estudio, donde se ubica el municipio de Mexicali y el condado de Imperial, el crecimiento de los casos positivos por las primeras 10 semanas es más notable en el municipio bajacaliforniano, aunque el condado californiano tiene un crecimiento muy acelerado que logra igualar las magnitudes tres semanas después y a la siguiente superar en casos a Mexicali. En las 7 semanas finales hay una disminución de casos en ambas ciudades.

El comportamiento temporal comentado puede ser mejor apreciado en la Figura 6 y Figura 7 para el corredor San Diego-Ensenada y San Diego-Mexicali donde se representan las tasas de contagio por cada 100000 habitantes que al estar relacionadas a la población permite su comparabilidad.

Las interrelaciones comentadas son apoyadas por el coeficiente de correlación de los valores observados. En la Figura 8 se puede apreciar cómo abajo a la derecha se muestra un bloque de correlaciones significativas en el que se incluye también la primera línea, este bloque está conformado por Tijuana, San Diego, Ensenada, Playas de Rosarito y Tecate donde las relaciones más fuertes son San Diego-Ensenada, San Diego-Playas de Rosarito y Tijuana-Tecate, no obstante, las relaciones espaciales implican que el acceso de San Diego al corredor turístico se haga a través de Tijuana. 
Figura 6. Comportamiento temporal de la tasa de contagio en el corredor San Diego-Ensenada
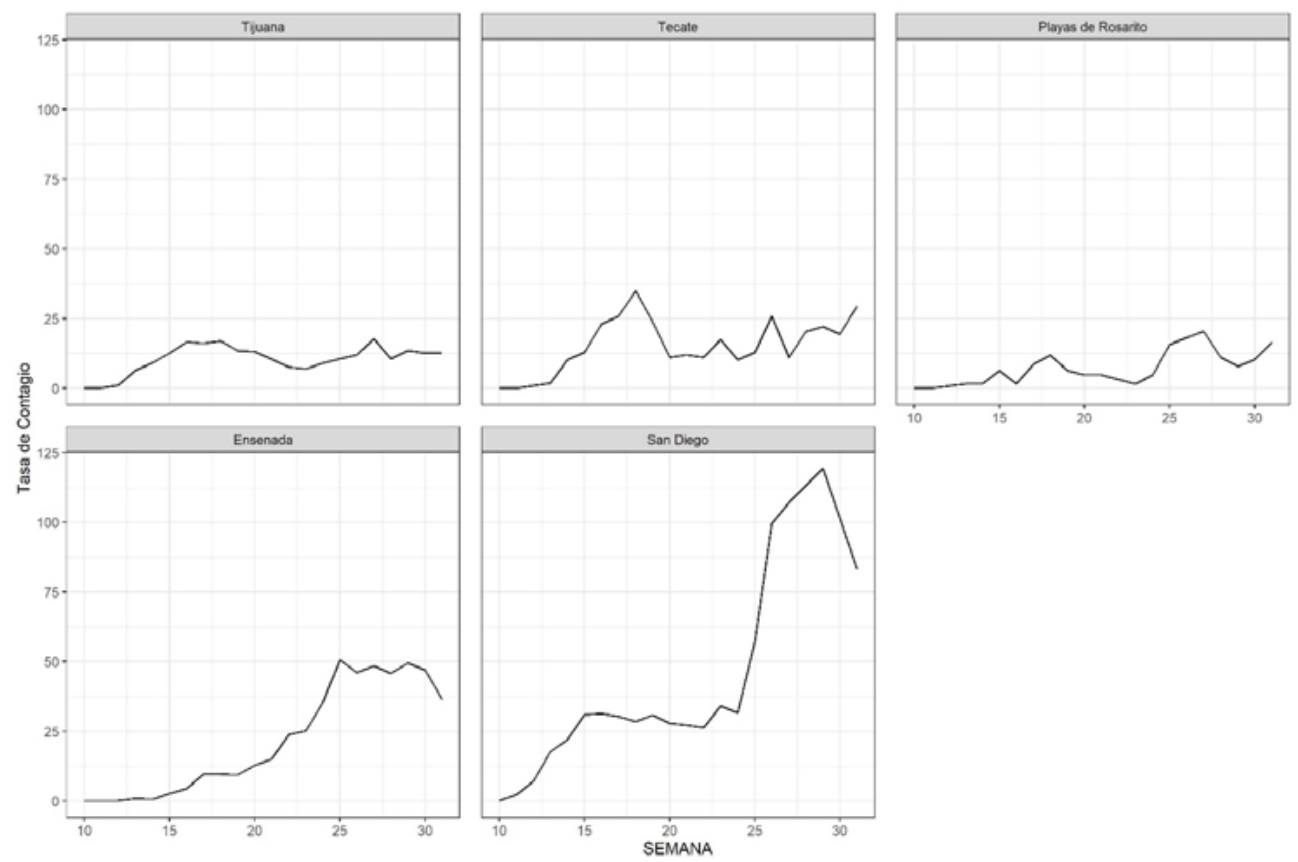

Fuente: Elaboración con base en estadísticas de la DGE-SS (2020) y de USAFacts.org (2020)

Figura 7. Comportamiento temporal de la tasa de contagio en el corredor San Diego-Mexicali
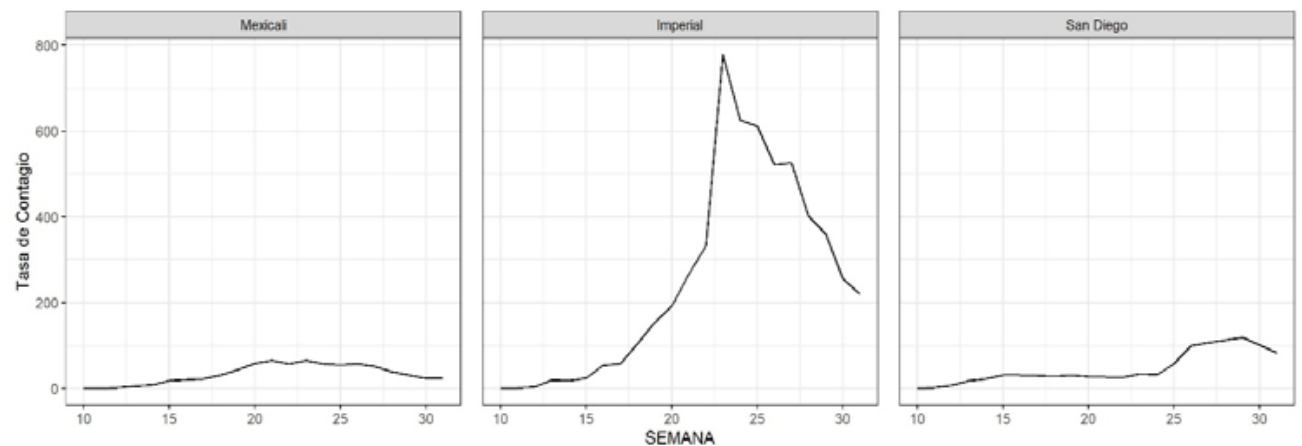

Fuente: Elaboración con base en estadísticas de la DGE-SS (2020) y de USAFacts.org (2020)

En adición, también hay otro bloque a la izquierda de la figura conformado por Mexicali, Imperial, San Diego, Ensenada y Playas de Rosarito, toda vez que la conectividad de infraestructura vial entre Mexicali y Ensenada o Playas de Rosarito puede significar una barrera importante en la concepción de la relación espacial entre ellas. Al considerar lo anterior, la interrelación puede limitarse al espacio local de Mexicali-Imperial que puede llegar a incluir a San Diego por el tamaño y diversidad de su economía. 
Figura 8. Coeficientes de correlación de los casos positivos por semana

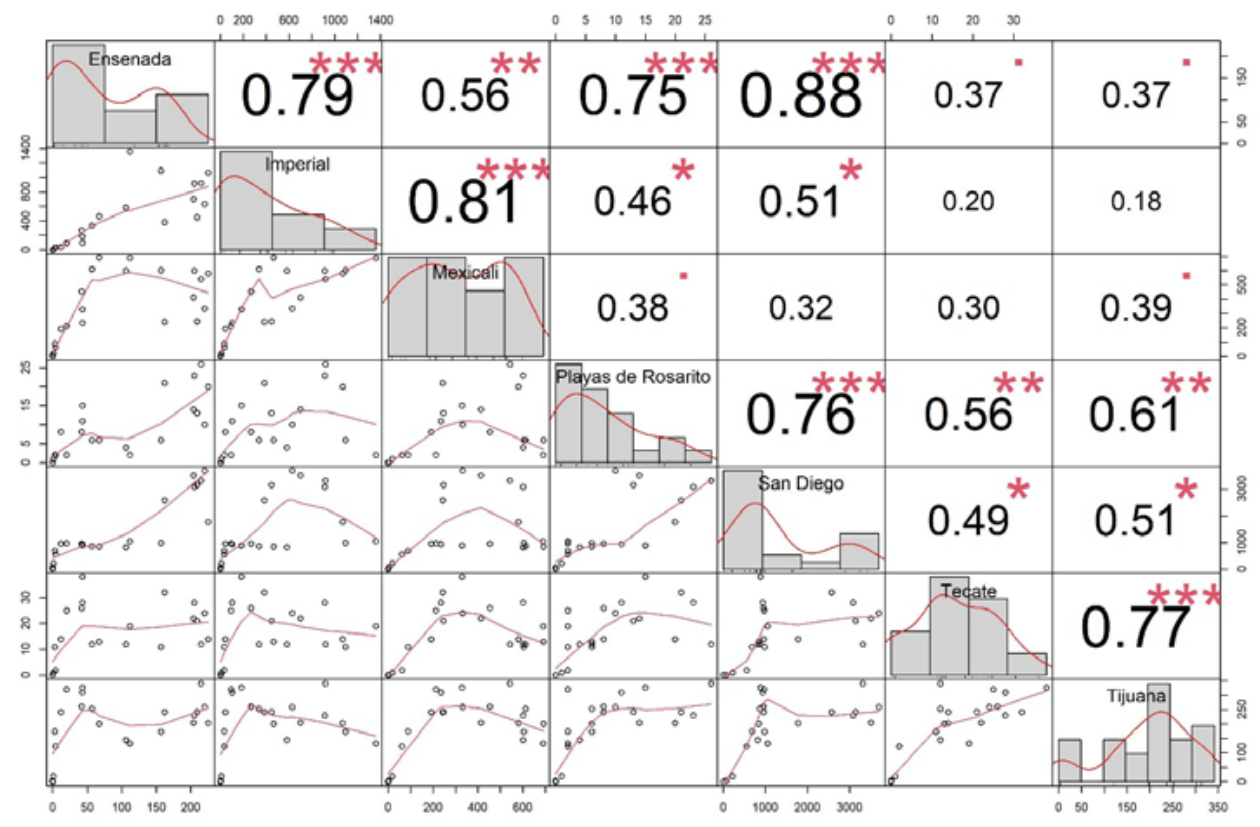

Fuente: Elaboración con base en estadísticas de la DGE-SS (2020) y de USAFacts.org (2020)

Los argumentos expuestos se fundamentan en la conocida primera ley de la geografía que establece que todas las cosas están correlacionadas entre sí, pero las cosas más próximas tienen una relación más fuerte que las más lejanas. Al considerar esto, se puede visualizar la relación espacio-temporal de la propagación del COVID en el área de estudio al utilizar una interpolación por el método de ponderación del inverso de la distancia (IDW) que supone que las similitudes entre los valores de los puntos de una superficie y la tasa de correlación entre ellos es inversamente proporcional a la distancia. Los resultados, para la cantidad de casos positivos en las semanas 20, 25 y 31 que se muestran en la Figura 9, dan cuenta de que en todo momento San Diego tiene la mayor cantidad de casos y que, además, tiene una dinámica creciente y que por su magnitud se manifiesta relativamente independiente; en Mexicali e Imperial la semana 20 muestra una dinámica asociada que se transforma a ser dominada por el incremento en Imperial y que hacia el final del periodo analizado es de menor visibilidad. En el caso de Tijuana, Tecate, Playas de Rosarito y Ensenada, la semana 20 muestra dinámicas diferenciadas con valores mayores en Tijuana que para la semana 25 tienen una apariencia de dinámicas asociadas y diferentes a las del resto y hacia el final del periodo lo que más destaca son la diferencias en las dinámicas de los municipios bajacalifornianos respecto a los condados de California.

$\mathrm{Al}$ considerar un análisis similar para la tasa de contagio por cada 100000 habitantes es notable la similitud de los valores para Tijuana, San Diego, Tecate, Playas de Rosarito y Ensenada y para Mexicali y Valle Imperial, aunque en estos últimos impactados de forma distinta dado el acelerado crecimiento de la tasa en el Valle Imperial que la variación de Mexicali, a pesar de que disminuye significativamente al final de las semanas estudiadas aún mantienen una diferencia relativa (Figura 10). 
Figura 9. Interpolación IDW de casos positivos en el área de estudio

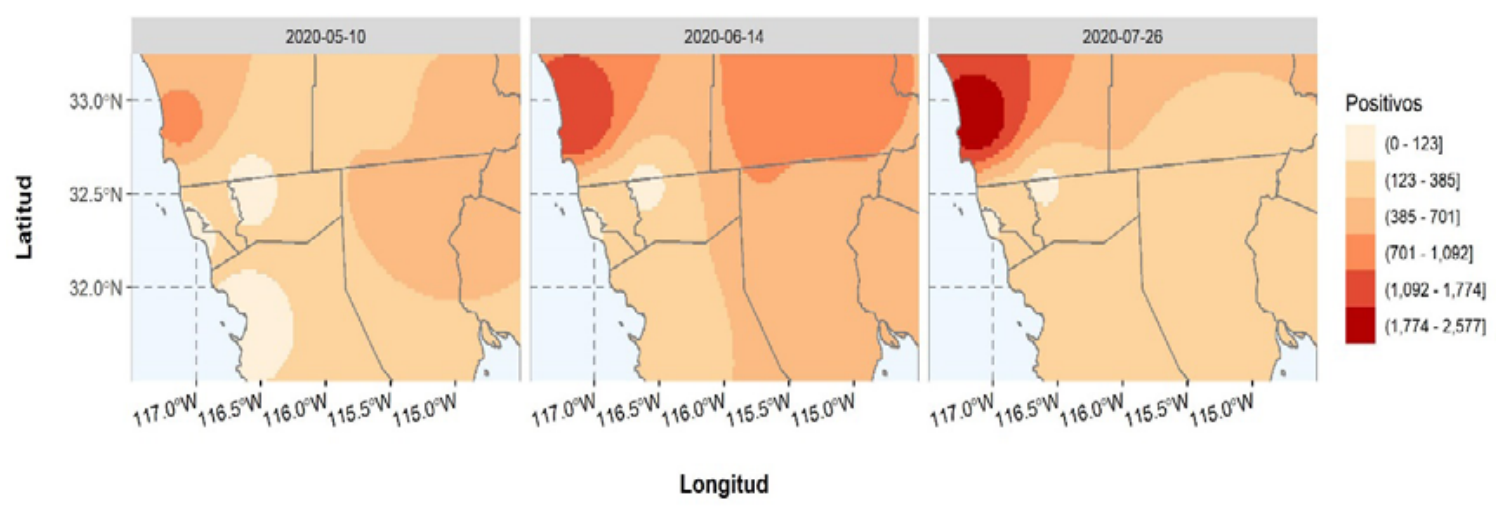

Fuente: Elaboración con base en estadísticas de la DGE-SS (2020) y de USAFacts.org (2020)

Figura 10. Interpolación IWD de tasa de contagio en el área de estudio
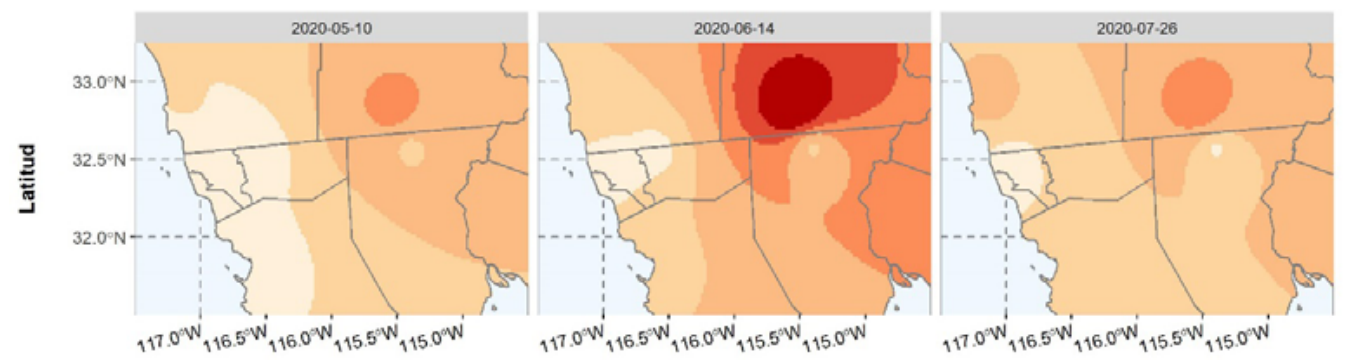

Tasa $\times 100,000$

$(0-26]$

$(26-66]$

(BB - 154]

$(154-266]$

$(266-402)$

$(402-626)$

Longitud

Fuente: Elaboración directa con base en estadísticas de la DGE-SS (2020) y de USAFacts.org (2020)

En síntesis, se confirma un patrón espacial que tiende a centralizarse en Mexicali y Tijuana, municipios donde residen los trabajadores transfronterizos que cotidianamente cruzan la línea internacional para laborar en alguno de los condados de California. Con una dinámica temporal cambiante hacia Ensenada y Playas de Rosarito, destino de la mayoría de los turistas californianos que recorren la ruta turística del corredor costero que va desde Ensenada hasta Tecate, y cruza los valles del vino en busca de las playas de Playas de Rosarito.

\section{Conclusiones}

En lo que respecta al patrón temporal epidemiológico de Baja California se destacan los municipios de Mexicali y Tijuana como los principales focos de contagio con desplazamiento hacia Ensenada, Playas de Rosarito y Tecate. Dicho patrón de contagios 
inicialmente muestra que los casos importados ocurren en municipios bajacalifornianos que albergan a los trabajadores transfronterizos que son personas que residen en estos municipios, pero que cotidianamente cruzan la línea fronteriza para trabajar en los condados de San Diego y Valle Imperial en California. Posteriormente, los contagios se desplazan hacia Ensenada, Playas de Rosarito y Tecate que son el destino de la mayoría de los turistas californianos que recorren la ruta turística del corredor costero. Lo anterior tiene implicaciones en la forma en cómo se ha administrado la epidemia y costos diferenciados para los habitantes en Baja California.

El patrón epidemiológico está influenciado por el tránsito fronterizo de trabajadores transfronterizos que tienen la doble nacionalidad que garantiza el cruce sin ningún tipo de control entre países, incluso cuando estos podrían estar infectados. Además de que los turistas estadounidenses durante todo este periodo fueron bienvenidos a visitar los atractivos turísticos del corredor costero bajacaliforniano, incluso cuando el número de contagios era casi ocho veces superior que el de los municipios del lado mexicano. Mientras, los residentes bajacalifornianos fueron limitados a transitar hacia California solo por actividades esenciales, como si la pandemia estuviera solo en el lado bajacaliforniano.

Por lo anterior, surge la necesidad de intervenciones de las autoridades de salud para controlar la epidemia no solo concentrándose en la detección inmediata de casos autóctonos sino mejorando la identificación de los casos importados con el fin de mitigar la propagación del virus en la entidad, así como de acuerdos internacionales sobre los controles sanitarios para que no haya un trato desigual entre los ciudadanos de uno y otro país.

\section{Referencias}

Bhaskar, A. Ponnuraja, C., Srinivasan, R. \& Padmanaban, S. (2020). Distribution and growth rate of COVID-19 outbreak in Tamil Nadu: A log-linear regression approach. Indian Journal of Public Health, 64(6), 188-191. https:/ / doi.org/10.4103/ ijph.ijph_502_20

Castro, C. A., Lodoño, L. A. \& Valdés, J. C. (2005). Modelación y simulación computacional usando sistemas de información geográfica con dinámica de sistemas a fenómenos epidemiológicos. Revista Facultad de Ingeniería Universidad de Antioquia, (34), 86-100. https://www.redalyc.org/articulo.oa?id=43003408

Cuartas, D., Arango-Londoño, D., Guzmán-Escarria, G., Muñoz, E., Caicedo, D., Ortega, D., Fandiño-Losada, A., Mena, J., Torres, M., Barrera, L. \& Méndez, F. (2020). Análisis espacio temporal del SARS-COV-2 en Cali, Colombia. Revista Salud Publica, 22(2), 1-6. https://doi.org/10.15446/rsap.v22n2.86431

Delgado, J. A. (2017). Dinámica de sistemas aplicado a la epidemiología. Seminario permanente: las matemáticas aplicadas a la epidemiología. Facultad de Matemática. Umc. Colombia. http://www.mat.ucm.es/ aramosol/TextoDeConferencia-JoseAlfonsoDelgado.pdf

Dirección General de Epidemiología de la Secretaría de Salud (DGE-ss). (2020). Bases de datos COVID 19 en México. https://www.gob.mx/salud/documentos/datos-abiertos- 152127 
Forrester, J. W. (1960). The impact of feedback control concepts on the management sciences. Foundation for Instrumentation Education and Research.

García Piñera, A. (2014). Modelos de ecuaciones diferenciales para la propagación de enfermedades infecciosas (trabajo de grado). Facultad de Ciencias. Universidad de Cantabria, España. https://repositorio.unican.es/xmlui/bitstream/handle/10902/7125/Andrea\%20Garcia\%20Pi\%C3\%B1era.pdf?sequence=1\&isAllowed=y

Kermack, W. O. \& McKendrick, A. G. (1927). A contribution to the mathematical theory of epidemics. Proceedings of the royal society of London. Series A, Containing papers of a mathematical and physical character, 115(772), 700-721. http://links. jstor.org/sici?sici=0950-1207\% 2819270801\%29115\%3A772\%3C700\%3AACTTMT\%3E2.0.CO\%3B2-Z

Medeiros, A., Daponte, A., Moreira, D., Gil-García, E. \& Kalache, A. (2020). Letalidad del COVID-19: Ausencia de patrón epidemiológico. Gaceta Sanitaria. Artículo en prensa. https://doi.org/10.1016/j.gaceta.2020.04.001

Miramontes, O. (2020). Entendamos el COVID-19 en México. COVID-19 en México. http://scifunam.fisica.unam.mx/mir/corona19/

Ortigoza, G., Lorandi, A. \& Neri, I. (2020). Simulación numérica y modelación matemática de la propagación del COVID-19 en el estado de Veracruz. Revista Mexicana de Medicina Forense y Ciencias de la Salud, 5(3), 21-37. https:/ / www.medigraphic.com/cgi-bin/new/resumen.cgi?IDARTICULO=94909

Palacios, B. (2020). Breve cronología de la pandemia 28 de febrero/14 de septiembre de 2020. Ibero, 70. http://revistas.ibero.mx/ibero/uploads/volumenes/55/ pdf/breve-cronologia-de-la-pandemia.pdf

Parr, J. (2020, 5 de mayo). COVID-19 data analysis, part 5: different models of infection rates in Mexico and what they tell us. Digital@DAI. https://dai-global-digital.com/covid-19-part-5-different-methods-to-model-infection-rates-in-mexicoand-what-they-tell-us.html

Pérez, V. (2012). Estrategia de vacunación para una epidemia de influenza (Tesis de maestría). сімат, México.

Ruiz, V. R. (2020). COVID-19 México, modelo matemático revela que sistema sanitario de México estará rebasado entre mayo y junio. Revista Contra Linea. https:// contralinea.com.mx/covid-19-modelo-matematico-revela-que-sistema-sanitario-de-mexico-estara-rebasado-entre-mayo-y-junio/

USAFacts.org (2020). US COVID-19 cases and deaths by state. https://usafacts.org/ visualizations/coronavirus-covid-19-spread-map

Vargas Magaña, R. M., Vargas Magaña, M. \& Fromenteau, S. (2020). Impacto de las medidas de control en la evolución del brote COVID-19. En colaboración con el Colectivo Científicos Mexicanos en el Extranjero. https://mexiciencia.github. io/ y Laboratorio ConCiencia Social https://concienciasocialla.wixsite.com/ misitio 
Alejandro Brugués Rodríguez

Mexicano, nacido en Ciudad de La Habana, Cuba. Doctor en ciencias económicas por la Universidad Autónoma de Baja California. Profesor-investigador del Departamento de Estudios Económicos de El Colegio de la Frontera Norte. Miembro del sNi nivel I. Líneas de investigación: estudios regionales y desarrollo económico. Publicación reciente: Fuentes, N. A., Brugués, A. \& Carrillo, J. (2020). Impacto económico de la reducción de la tasa del iva en la región fronteriza norte de México con base en el uso de precios implícitos en el modelo insumo-producto. Revista de economía, 37(95).

\section{Noé Arón Fuentes Flores}

Doctor en economía por la Universidad de California, Irvine. Profesor investigador del Departamento de Estudios Económicos de El Colegio de la Frontera Norte. Miembro del SNI nivel II. Líneas de investigación: desarrollo regional, economía fronteriza y modelos Insumo-Producto. Publicación reciente: Fuentes, N. A., Brugués, A., González, G. \& Carrillo, J. (2020). El impacto económico en la industria maquiladora y en la región fronteriza del norte de México debido al alza de 100\% del salario mínimo. Región y sociedad, 32.

Alejandra Ramírez Cervantes

Licenciada en Economía por la Universidad Autónoma de Baja California. Líneas de investigación: educación financiera, innovación social, acceso a la información pública y protección de datos personales. 